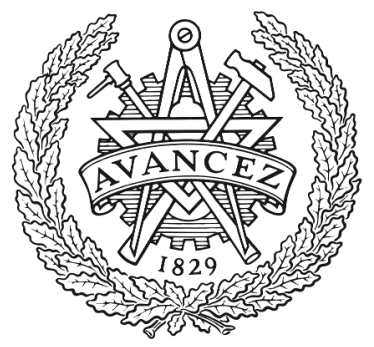

CHALMERS

UNIVERSITY OF TECHNOLOGY

\title{
Investigation of vehicle ride height and wheel position influence on the aerodynamic forces of ground vehicles
}

Downloaded from: https://research.chalmers.se, 2023-04-26 06:33 UTC

Citation for the original published paper (version of record):

Vdovin, A., Sebben, S., Walker, T. et al (2014). Investigation of vehicle ride height and wheel position influence on the aerodynamic forces of ground vehicles. International Vehicle Aerodynamics Conference: 81-90

N.B. When citing this work, cite the original published paper. 
This is a preprint version of the following paper:

Vdovin, Alexey \& Löfdahl, Lennart \& Sebben, Simone \& Walker, T., Investigation of vehicle ride height and wheel position influence on the aerodynamic forces of ground vehicles, The International Vehicle Aerodynamics Conference 2014, Pages 81-90 (2014), https://doi.org/10.1533/9780081002452.3.81.

Being a preprint and not the final accepted manuscript, this version does not include the corrections made to the paper after the review process and therefore it is slightly different from the published journal article. The readers are advised to follow the DOI link to access the published version online.

(C) 2018. This manuscript version is made available under the CC-BY-NC-ND 4.0 license http://creativecommons.org/licenses/by-nc-nd/4.0/

\title{
Investigation of vehicle ride height and wheel position influence on the aerodynamic forces of ground vehicles
}

\author{
A. Vdovin, L. Löfdahl $*$ \\ S. Sebben, T. Walker $* *$
}

*Applied Mechanics, Chalmers University of Technology, Sweden

$* *$ Volvo Car Corporation, Sweden

ABSTRACT

To prevent test vehicles from movement during experiments in modern aerodynamic wind tunnels, fastening struts are typically used for a rigid connection between the model and the force balance underneath the wind tunnel floor. A weakness of this experimental set-up is that such struts limit the vertical movement of the vehicle.

By analysing experimental data from the Volvo Cars wind tunnel and corresponding CFD simulations the differences in measurements using struts with and without vertical displacement have been analysed and compared. The model used was a Volvo S60.

\section{INTRODUCTION}

When using aerodynamic wind tunnels for road vehicle testing, it is generally accepted today that having a moving ground simulation system is essential for obtaining reliable results $[1,2,3]$. Common systems designed for passenger vehicle testing usually rely on single or multiple belts moving under the vehicle to represent the boundary conditions of driving on the road. To ensure model positioning in the wind tunnel, fastening struts that rigidly connect the test object to a force balance underneath the wind tunnel are usually used. One drawback of such a set-up is that together with lateral and horizontal movement, vertical displacement of the vehicle body is prevented. Under real road conditions, such vertical movement occurs due to the radial expansion of the tyres because of inertial forces, and due to the aerodynamic lift forces acting on the body of the vehicle.

This study investigates the magnitude of potential errors that could be introduced by having constant vehicle ride height when conducting tests in the wind tunnel at different velocity intervals. By comparing the data obtained using floating struts, that allow vertical displacement, with results acquired using completely fixed struts, the differences in the aerodynamic force coefficients due to changes in vehicle velocity are investigated.

\section{METHODOLOGY}

There are two main methods commonly used for assessing the aerodynamic design of passenger vehicles: wind tunnel testing and numerical CFD simulations. For this investigation both of those methods were used. First, a full-size production sedan type vehicle was tested in the Volvo Cars aerodynamic wind tunnel and later a model of the same vehicle was studied by means of CFD simulations. In this chapter the set-ups of the two different methods are presented. 


\subsection{Experimental set-up}

The vehicle chosen for this investigation was a Volvo S60 equipped with 215/50 ZR17 tyres, see Figure 1 . The wind tunnel used was a full-scale closed-circuit aerodynamic wind tunnel with a cross sectional area of $27 \mathrm{~m}^{2}$ and slotted wall inserts [4]. It was equipped with a five-belt moving ground system, installed on a turntable. This system consisted of a centre belt and four wheel drive units (WDU) rotating the wheels. The vehicle was held in position by struts that connected it to the force balance located under the test section.

For the experiment, two different sets of struts were used: fixed and floating. Fixed struts rigidly connect the vehicle to the balance; therefore the vehicle ride height could be set and kept constant during the experiment. Using such struts allowed to have a reduced load on WDUs since part of the vehicle weight was supported by the struts. Floating struts, on the other hand, prevent only lateral and longitudinal displacements of the test object, allowing vertical movement. Since on a real road the vehicle ride height is changing with speed, using floating struts may seem preferable, but the drawback of using this method is much higher loads on the WDUs, which is undesirable, hence in most of the cases fixed struts are used.
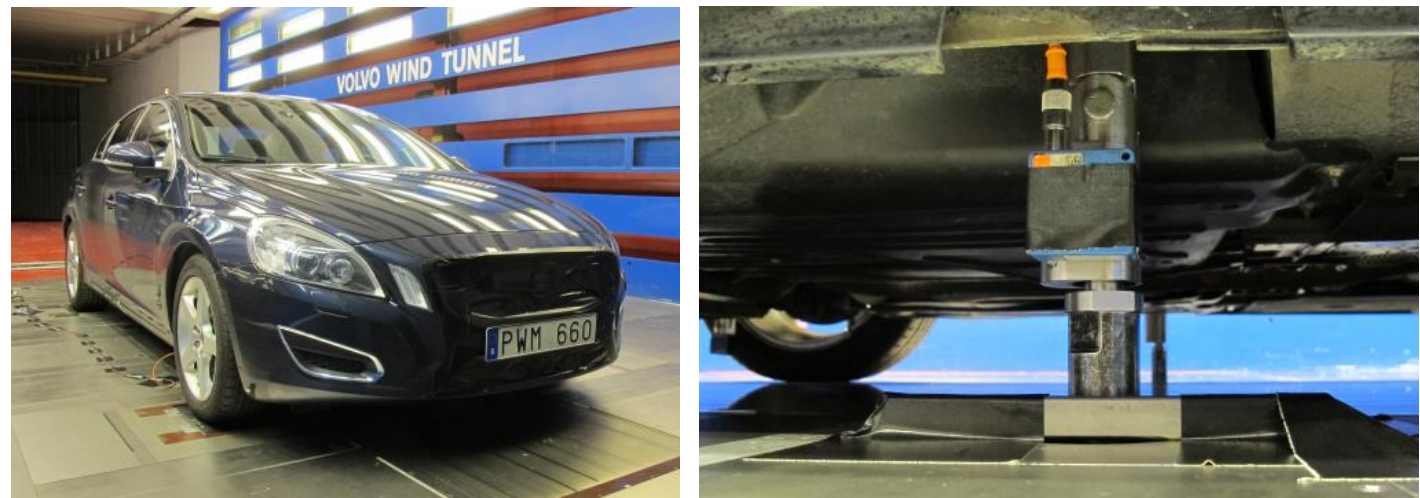

Figure 1. Overview of the wind tunnel model and displacement transducer attachment to the moving part of the floating strut.

Since one of the objectives of this investigation was to study the changes occurring to tyre shape and ride height with increasing velocity, it was decided to use a set of laser displacement transducers. The transducers used had a working range of $50-100 \mathrm{~mm}$ and an uncertainty of less than $0.1 \%$. The displacement was measured for front and rear suspension arms and, when using floating struts, for the vehicle body displacement. An overview of the test set-up and example of the transducer installation can be seen in Figure 1. It should also be mentioned that the initial trim heights for front and rear of the vehicle were adjusted to be exactly the same between different tests and matching the specifications. In case of floating struts, that was achieved by loading the vehicle with an appropriate number of sand bags.

Previous investigations have shown significant tyre shape changes with increasing speed $[5,6]$. The rotating wheel is subjected to distributed inertial force; consequently there is radial expansion and axial contraction of the tyre. This force also leads to wheel centre height increase and tyre shape changes in the contact patch area.

The tyre shape changes observed previously were measured for fixed struts only and for a different vehicle/wheels combination. Hence, in order to investigate the level of deformation for the tyres used in the experiment and its dependency on different strut designs, the front right tyre was filmed from different angles during the separate set of tests.

The wind tunnel force balance was used to measure aerodynamic forces acting on the vehicle body in the range of velocities from $80 \mathrm{~km} / \mathrm{h}$ to $240 \mathrm{~km} / \mathrm{h}$. The cases investigated included different runs with fixed and floating struts. In some of the cases the frontal cooling inlets were covered with adhesive tape to investigate the effects of cooling flow and estimate the cooling drag. Additionally, a number of tests were conducted without wind, to measure the relative contribution of aerodynamic forces to tyre deformation and vehicle ride height.

\subsection{Numerical set-up}

For the numerical part of the investigation the corresponding vehicle model was prepared. Commercial preprocessing software ANSA v14.2.1 was used for cleaning part geometries, putting them together and closing multiple gaps. The vehicle ride height and pitch angle were adjusted to match the specifications used in the wind tunnel. Suspension geometry and the engine compartment were represented with a high level of accuracy.

Even though previous investigations had shown the importance of tyre pattern for CFD simulations [7], due to computer resources limitation and for repeatability reasons it was decided to use treadles (slick) tyre models. However, these models had production profiles obtained from the tyre manufacturers. For the different cases investigated, the tyre models were morphed to correspond to the shapes observed during the wind tunnel tests [5]. It should be noted that, although the tyre shape was altered, the contact patch was kept the same as in static conditions. The influence of this parameter was not quantified. 
As a part of the preparation process some interface surfaces were added to the model in order to separate regions with different physics specifications or for different volume mesh type desired.

The simulations and post-processing were conducted in the commercial CFD software StarCCM+ v9. The numerical method used in this investigation was a standard automotive CFD procedure based on guidelines from the Volvo aerodynamic calculation practice. The steady-state approach was used in RANS (Reynolds-Averaged Navier-Stokes) mode with the k-epsilon realizable turbulence model and coupled solver. The maximum surface cell size used was $10 \mathrm{~mm}$ with an automatic refinement down to $1 \mathrm{~mm}$ on sharp edges and where it was required. In order to properly resolve the boundary layer and maintain y+ values in the range between 30 and 150 [8], the volume mesh generated contained 3 prism layers on all surfaces, except the engine compartment, with a total height of $7 \mathrm{~mm}$. The mesh generated was mostly hexahedral with refinement zones for the engine bay, underbody, wheel-houses, mirrors and base wake areas.

The components of the cooling package were represented by separate porous regions with corresponding inertial and viscous tensors specified to ensure realistic pressure drop and air flow through them. To simulate the rotation of the wheels moving wall boundary condition was used with MRF (multiple reference frame) regions between spokes. The same method was used to model rotation of the two cooling fans.

The numerical wind tunnel created was very simplified and had large enough dimensions not to result in any significant blockage or interference effects. The boundary conditions used were typical for such type of simulations: velocity-inlet, pressure outlet, moving wall for the ground and symmetry boundary condition for all walls and the ceiling. Since the same procedure was used for meshing different cases the final volume mesh for each case was around 95 million cells.

Testing models in CFD with varying velocities requires changing the volume mesh, especially in the boundary layer next to the vehicle surface. In order to minimise possible influences of mesh dependencies, it was assumed in this investigation that aerodynamic coefficients were independent of wind velocity and the only changes occurring to them resulted from the geometrical changes of the model. Hence, boundary conditions were maintained constant and corresponding to $100 \mathrm{~km} / \mathrm{h}$ road velocity for all cases investigated. Only the geometrical shape of tyres and positioning of the vehicle body in space were being changed.

\section{RESULTS \& DISCUSSIONS}

In this chapter the main results of the investigation are presented and discussed. Firstly, the observed and measured tyre deformations and vehicle body displacements are presented, and later the changes in aerodynamic forces that were measured by the wind tunnel balance and computed in CFD are compared.

\subsection{Tyre geometry change}

As discussed in earlier works [5], a rotating tyre experiences a set of deformations due to the presence of distributed inertial force: radial expansion, axial contraction and wheel centre height lift, see Figure 2 for the definitions.

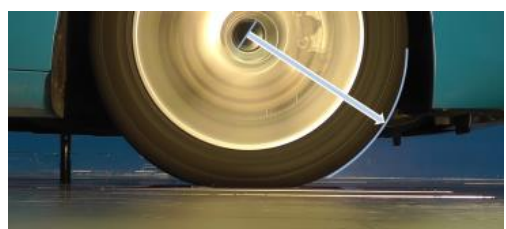

(a)

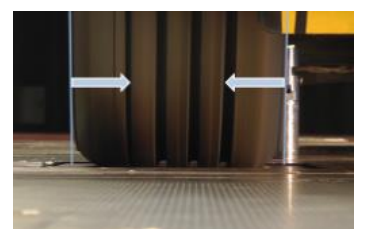

(b)

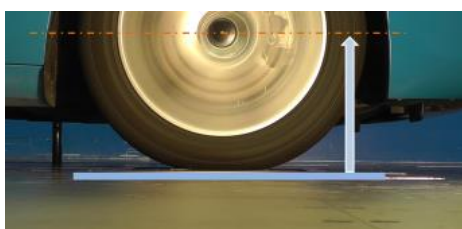

(c)

Figure 2. Definitions for the tyre deformations: (a) radial expansion; (b) axial compression; (c) vertical lift.

By analysing the video footage of the rotating tyre it was found that radial expansion and axial compression of the tyres were almost identical for the cases of fixed and floating struts. The values measured for the front right wheel that were later used for tyre morphing with the trend-line added to them can be found in Figure 3 . As one can see, both radial expansion and axial compression show quadratic relationship, as previously suggested [5].
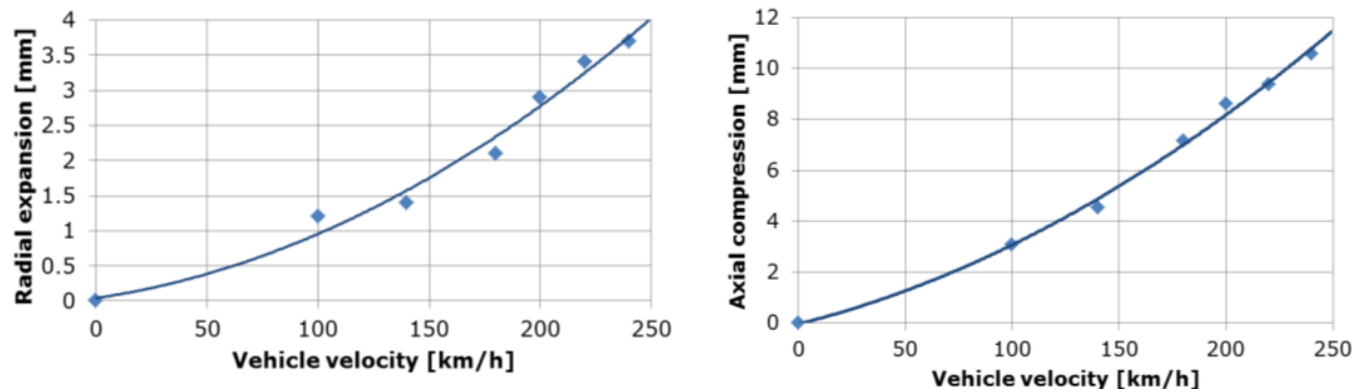


\section{Figure 3. Radial expansion and axial compression of tyres}

The most significant difference in tyre deformation between cases with different sets of struts was the wheel centre lift. In Figure 4 one can see the values for front wheels measured by the laser transducers under normal wind tunnel conditions. It is clear that floating struts allow significantly higher vertical displacement of the wheel centre. This happens mostly because of two factors. Firstly, the initial wheel centre height for the floating struts case was lower, because the wheels were supporting the full weight of the vehicle, unlike the fixed struts case where part of the weight was taken by the struts. Secondly, in the case of floating struts the vehicle ride height increased with speed, as discussed in part 3.2, and therefore the suspension loads were kept almost the same. On the other hand in case of fixed struts, as the wheel centre lifted, the suspension springs were being loaded and the force counteracting the lift increased.

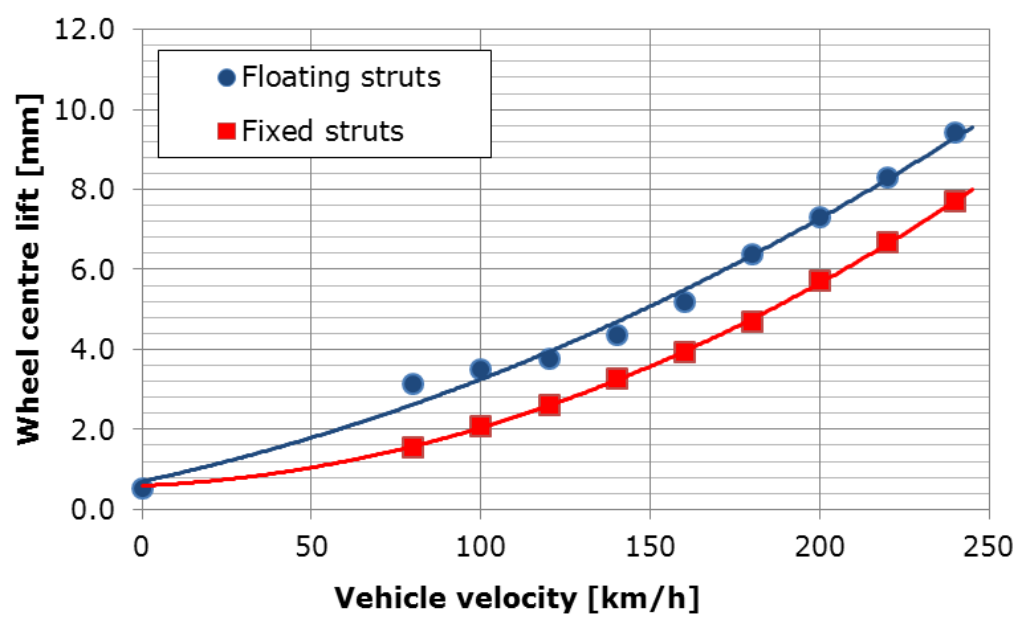

Figure 4. Wheel centre lift of tyres depending on the velocity for the different struts used

It should also be noted that one of the tests conducted with floating struts included a velocity sweep without wind (just wheels rotating), which allowed the study of the relative contribution of aerodynamic lift forces acting on a vehicle body to the wheel centre lift. As expected the main contributors to wheel vertical displacement are distributed inertial forces, but the impact of aerodynamic lift of the vehicle body can also be rather considerable. The lift forces acting on the vehicle body caused it to rise, lowering vertical load transferred through the wheels. It was especially important for the rear part of the vehicle where the contribution of aerodynamic forces to wheel centre lift was as high as $1.5 \mathrm{~mm}$.

Another important issue that was observed during the experiment was hysteresis in the height measurements, meaning that the tyres and the vehicle did not return to their initial state after the full cycle of the velocity sweep. Even when fully stopped there were always some residual deformations left. An example of that can be seen in Figure 4, where the initial lift measured for the wheels is a little higher than zero. This effect has been previously noticed [9] and it is assumed to be caused by temperature dependency of the tyre characteristics and changing air pressure inside the tyre. The maximum residual wheel centre lift observed was as high as $2 \mathrm{~mm}$. This can be an important factor to consider during multiple velocity sweeps without much time in between them.

\subsection{Vehicle body positioning change}

By definition, fixed struts prevent any vehicle body movement during the test, therefore in this part only the floating struts will be considered. As discussed in section 2.1, two laser displacement transducers were installed on the moving parts of floating struts. Then, the measurements obtained were used for calculating the displacements of the vehicle body at the positions of front and rear suspension; a simple linear approximation was applied. These displacements will be further referred to as front and rear body lifts. In Figure 5, one can see the changes of these parameters with speed for two cases: wind on and wind off. 


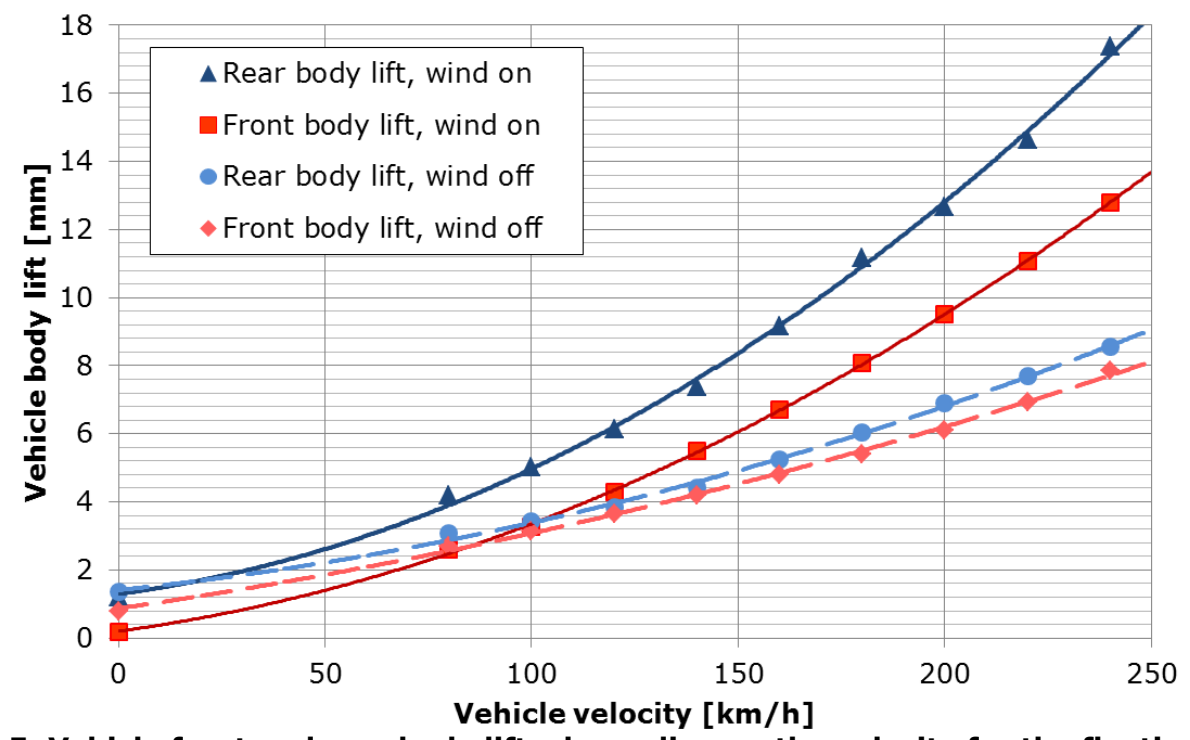

Figure 5. Vehicle front and rear body lifts depending on the velocity for the floating struts

As expected, in the case of no wind, front and rear body displacements directly match the wheel centre lifts of the front and rear wheels. In case of wind on, with aerodynamic lift forces acting on the vehicle body, the vehicle position in space experienced quite significant changes. As can be seen from Figure 5 , at the maximum velocity the front of the vehicle rose almost by $13 \mathrm{~mm}$ and the rear by nearly $18 \mathrm{~mm}$. The difference may be explained by the fact that lift forces, acting on front and rear of the vehicle are not equal, as well as weight distribution and suspension stiffnesses. The aerodynamic lift forces and the resulting vertical displacements may vary significantly based on the design of the vehicle in general and even shape of such small parts like wheel rims [9].

Figure 5 shows again some residual displacements at low velocities, since the tyres did not have time to cool down completely to the ambient temperature after the previous tests.

\subsection{Aerodynamic forces}

In this part aerodynamic drag and lift forces are discussed as well as cooling drag. Aerodynamic resistance moments acting on rotating wheels, which can be affected by the changing geometry of the tyres were not considered, since measuring these moments required significant changes in the wind tunnel set-up [10].

\subsubsection{Aerodynamic drag force}

Figure 6 shows the aerodynamic drag coefficient development with changing velocity measured in the wind tunnel and by means of CFD simulation. Both graphs use $80 \mathrm{~km} / \mathrm{h}$ fixed struts case as a reference.
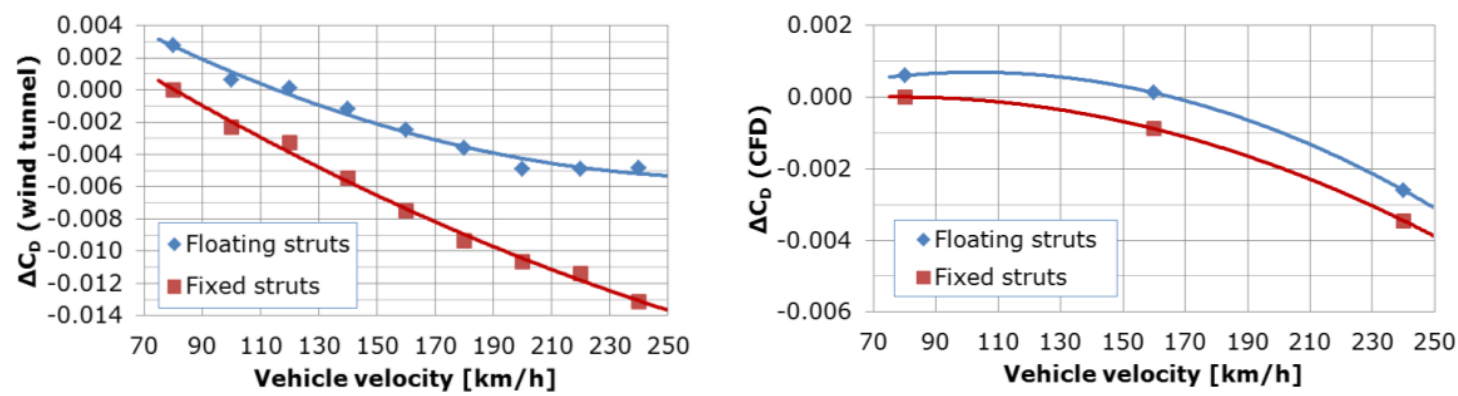

Figure 6. Drag coefficient behaviour in relation to velocity for wind tunnel tests and CFD simulations

As one can see, aerodynamic drag coefficient decreased with speed. This behaviour has already been observed for the case of fixed struts [6]. Floating struts also demonstrated a drop in measured drag coefficient with speed, though this drop was lower in magnitude. Moreover for the case of floating struts the $C_{D}$ appears to stabilize around $200 \mathrm{~km} / \mathrm{h}$, whereas for the fixed struts $C_{D}$ continues to drop. One of the reasons for this difference observed between the two cases may be the fact that in the case of fixed struts, expanding tyres moving up inside wheel-house change the flow field inside and especially outside of the wheel-house significantly, hence, affecting the wheel wakes. In addition, the exposed area of the tyres is reduced. This is not the case for floating struts, where the vehicle body rises together with the wheel centres, resulting in slightly increased rear wake.

Another interesting observation that can be made here is that the drag measured with floating struts was always higher in comparison with fixed struts measurement (at corresponding velocities). Based on the wind tunnel results the difference in measurement could be as high as 8 drag counts. This was most likely a consequence of significant vehicle body lift, especially in the rear part, that affected the base wake of the vehicle. 
CFD results follow the same decreasing trend as the experimental ones; however, the magnitude of the changes was less significant. It should be mentioned that in addition to the previously mentioned simplifications in terms of using slick tyres and fixed geometry of the contact patch area, the suspension of the vehicle was also kept unchanged. An extension of this analysis may be to investigate the effects of the simplifications mentioned and also to increase cell resolution for the underbody as the standard CFD procedure used may have been not refined enough to capture the effects of such geometrical changes. It may also be interesting to investigate the influence of the struts geometry since they were not modelled in the CFD simulations.

\subsubsection{Aerodynamic lift forces}

As the ride height of vehicle is changing when using floating struts, more air is allowed to pass underneath the body which leads to some important changes in aerodynamic lift force distribution. The difference between lift coefficients for cases of floating and fixed struts are presented in Figure 7.

Figure 7 shows that the total lift coefficient for the case of floating struts was always higher than for fixed struts. Curves for front and rear lift coefficients demonstrate much more complicated differences between the two cases. One can see that substantial rear lift of the vehicle at high speeds observed in the case of floating struts is linked to higher lift force acting on it.

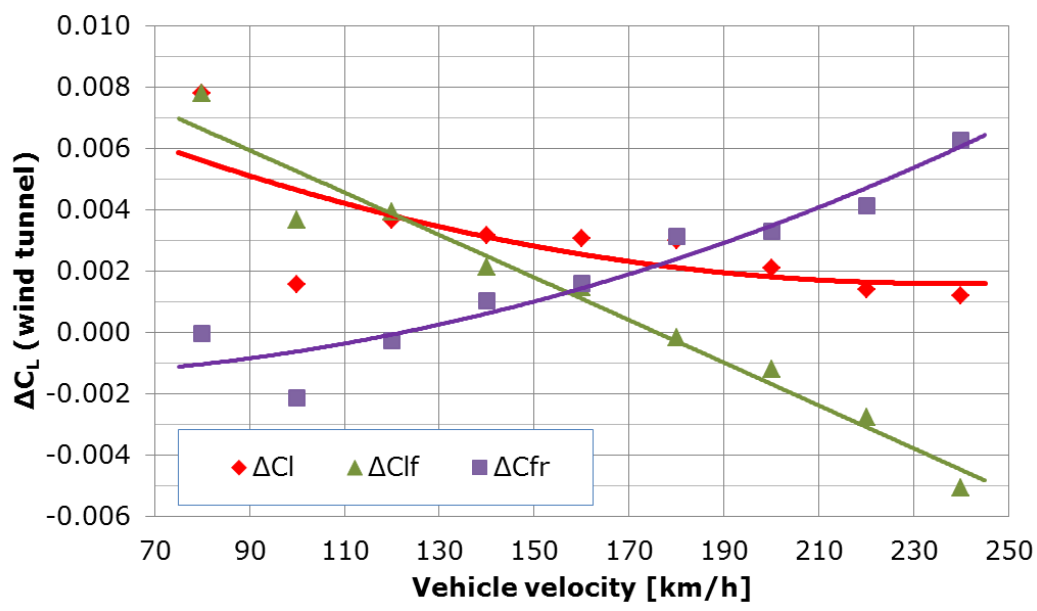

Figure 7. The difference in lift coefficients (total, front, rear) between cases of floating and fixed struts

\subsubsection{Cooling drag}

In the wind tunnel tests the cooling drag was defined by the difference between aerodynamic drag coefficients of a model with an open front grill and a model with covered grill that did not allow any airflow through the front of the vehicle. Figure 8 shows the cooling drag values derived from the wind tunnel tests data.

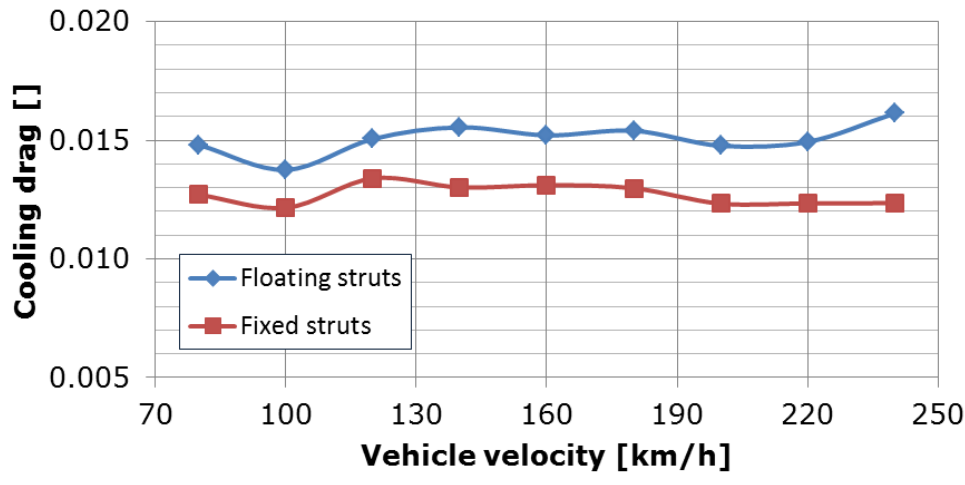

Figure 8. Cooling drag dependency on the velocity for two sets of struts

As can be seen, the cooling drag measured for the floating struts case was always higher than for the case of fixed struts. This difference can be as high as 4 drag counts for higher velocities where a sharper distinction between two cases is achieved. CFD simulations did not show any considerable difference in the air mass flow through the cooling system, therefore it is expected that the variation in cooling drag was a result of changing interference between cooling air and external flow.

\section{CONCLUSIONS}

It is a well-known fact that the tyre shape changes with increasing velocity. This investigation has shown that the tyre shape also depends on the type of struts used for connecting the vehicle body to the force balance. 
Tyre deformations depending on the vehicle velocity for the cases of fixed and floating struts were measured. The floating struts allow displacement in the vertical direction, which leads to higher wheel centre positions, and most importantly to substantial body lift, especially for the rear part of the vehicle. The lift of the wheel centres and vehicle body were measured using laser displacement transducers and presented. In comparison to the difference in wheel centre lifts, the differences in radial expansion and axial compression of the tyres were insignificant.

Experimental and numerical tests have shown that there were substantial differences in aerodynamic drag and lift forces acting on the vehicle for the two sets of struts that were investigated. Firstly, it has been shown that as a result of geometrical changes in tyres and suspension, and independently of the struts used, the drag coefficient decreased with speed. The drop was more significant for the case of fixed struts, resulting in 13 drag counts reduction between tests for $80 \mathrm{~km} / \mathrm{h}$ and $240 \mathrm{~km} / \mathrm{h}$. For the floating struts a drop of 8 drag counts was measured. Secondly, an important observation was made that aerodynamic drag coefficient was always higher if floating struts were used. The difference increases with speed and can be as high as 8 drag counts. Numerical simulations confirmed both trends discussed, though it should be noted that the magnitude of differences estimated by CFD was smaller. A number of reasons for this have been suggested and have to be further investigated.

Comparing total aerodynamic lift coefficients, the values for the case of floating struts were always somewhat higher, with the maximum difference observed at $80 \mathrm{~km} / \mathrm{h}$ where the difference measured was as high as 8 lift counts. It has also been shown that in the case of floating struts there is a slight shift of lift forces towards the rear of the vehicle, meaning a reduction for front lift coefficient and corresponding increase for the rear one.

To conclude, it should be stated that even though traditionally aerodynamic coefficients for passenger vehicles are considered to be Reynolds independent, this assumption should be treated with caution. The geometrical changes in terms of tyre deformations and vehicle body lift, as shown in this investigation, may result in significant alteration of aerodynamic forces.

\section{REFERENCE LIST}

[1] J. Wiedermann, "The Influence of Ground Simulation and Wheel Rotation on Aerodynamic Drag Optimization - Potential for Reducing Fuel Consumption," in SAE paper No.960672, 1996.

[2] E. Mercker, N. Breuer, H. Berneburg and H. J. Emmelmann, "On the Aerodynamic Interference Due to the Rolling Wheels of Passenger Cars," in SAE International (Paper No: 910311), Warrendale, PA, 1991.

[3] P. Elofsson and M. Bannister, "Drag Reduction Mechanisms Due to Moving Ground and Wheel Rotation in Passenger Cars," in SAE Paper No. 2002-01-0531, SAE World Congress, 2002.

[4] J. Sternéus, T. Walker and T. Bender, "Upgrade of the Volvo Cars Aerodynamic Wind Tunnel," in SAE Technical Paper 2007-01-1043, 2007.

[5] P. Mlinaric and S. Sebben, "Investigation of the Influence of Tyre Deflection and Tyre Contact Patch on CFD Predictions of Aerodynamic Forces on a Passenger Car," in MIRA International Conference on Vehicle Aerodynamics, Coventry, England, 2008.

[6] C. Landstrom, L. Josefsson, T. Walker and L. Lofdahl, "Aerodynamic Effects of Different Tire Models on a Sedan Type Passenger Car," SAE Int. J. Passeng. Cars - Mech. Syst., vol. 5, no. 1, pp. 136-151, 2012.

[7] T. Hobeika, S. Sebben and C. Landstrom, "Investigation of the Influence of Tyre Geometry on the Aerodynamics of Passenger Cars," SAE Int. J. Passeng. Cars - Mech. Syst., vol. 6(1), no. doi:10.4271/2013-01-0955., pp. 316-325, 2013.

[8] CD-Adapco, StarCCM+ v8.04 manual -> Modelling physics -> Modelling turbulence and transition -> Using Wall treatment Models -> How do I decide on a wall treatment?, 2013.

[9] B. Schnepf, G. Tesch and T. Indinger, "On the Influence of Ride Height Changes on the Aerodynamic Performance of Wheel Designs," in JSAE Annual Congress, 2014.

[10] A. Vdovin, S. Bonitz, C. Landstrom and L. Lofdahl, "Investigation of Wheel Ventilation-Drag using a Modular Wheel Design Concept," SAE Int. J. Passeng. Cars - Mech. Syst., vol. 6, no. 1, 2013. 